\title{
ENSEÑANZA DEL ESPAÑOL CON FINES ESPECÍFICOS (EFE) Y SU APLICACIÓN A LA CIENCIA Y A LA TECNOLOGÍA
}

\author{
ISABEL SERRA PFENNIG
}

Universidad Politécnica de Madrid, España

\begin{abstract}
Resumen. El español y la cultura hispánica siempre han suscitado un gran interés en un público muy amplio, pero en los últimos años esta tendencia se ha desarrollado de forma muy notable en las universidades europeas no solo en el ámbito de las humanidades sino también en el resto de centros de educación superior. El español con fines específicos ha llegado a ser una asignatura opcional o de competencias en muchos centros de enseñanzas científicas o técnicas, como son las universidades politécnicas donde el español cumple la función de lengua para fines específicos y cada vez más es considerada una lengua de comunicación en el ámbito académico y laboral. Nuestro trabajo versa sobre dos puntos temáticos: por una parte abordamos la demanda del español con fines específicos (EFE) como instrumento de comunicación internacional en el marco tanto académico como profesional y su aplicación a la ciencia y a la tecnología. Por otra parte, la arquitectura y la construcción, como otras ciencias, constituyen por sí mismas un producto fundamental que engloba la industria, la tecnología y el conocimiento. Para ello, daremos a conocer algunos aspectos y recursos léxico-terminológicos en relación a la arquitectura y a la construcción, así como otras características del vocabulario científico-técnico.
\end{abstract}

Palabras clave: español con fines específicos, ciencia, tecnología, arquitectura y construcción

\section{INTRODUCCIÓN}

El objetivo del presente trabajo es por una parte, afianzar la importancia del español como lengua extranjera (ELE) como herramienta de comunicación y de transmisión de conocimiento tanto en un determinado contexto cultural (la historia del arte, las bellas artes etc.), así como en el mundo académico y profesional. Y por otra, proporcionar al estudiante o al profesional de la Arquitectura y de la Construcción las herramientas léxicas y terminológicas necesarias a partir de fuentes bibliográficas innovadoras y de carácter divulgativo y que sirvan de ayuda para entender mejor las distintas disciplinas que componen la arquitectura y la construcción. 
Por un lado, y en general, hemos procurado prestar especial atención a algunas publicaciones teóricas en torno a la enseñanza-aprendizaje del español con fines específicos (EFE), y por otro, y en particular, presentamos un manual de Arquitectura y Construcción como herramienta de enseñanza- aprendizaje que ayude al estudiante y al profesional a defenderse ante situaciones reales y comunicativas de aprendizaje del español en el ámbito de la ciencia y la tecnología. Por último, aportamos algunos ejemplos del léxico técnico con carácter divulgativo de los principales elementos de la arquitectura y de la construcción con su correspondiente traducción al inglés, francés alemán e italiano.

\section{EL ESPAÑOL COMO LENGUA EXTRANJERA (ELE). REFLEXIONES TEÓRICAS}

Durante las últimas décadas, la enseñanza del español como lengua extranjera se ha ido posicionando cada vez con mayor intensidad en los centros de enseñanza de educación superior y en mayor medida en las escuelas técnicas superiores de nuestro país. Esta demanda se debe no sólo a los procesos metodológicos y lingüísticos que han favorecido el interés por el aprendizaje del español, sino también por el hecho de los enormes cambios económicos y sociales de las últimas décadas y que han repercutido de manera notable en este acontecimiento. Recientemente, hemos observado un aumento cuantitativo de la demanda del español en las universidades politécnicas. Esto se debe por una parte, y sobre todo a nivel global, al interés creciente por el español sobre todo en grupos de estudiantes con necesidades lingüísticas muy diversas y según qué situación o contexto necesiten el uso del español. Y por otra, para que el estudiante pueda superar el nivel de acreditación exigida por parte de la Unión Europea para poder cursar sin trabas lingüísticas la lengua y facilitarle un nivel adecuado para su aprendizaje. Los teóricos de la comunicación coinciden también en la fuerte demanda del español en los últimos años y la necesidad de proporcionar al estudiante la formación lingüística-pragmática que le permita avanzar tanto en un desarrollo académico así como también profesional. Considerando la enorme diversificación tanto de los estudiantes como de los contextos, las situaciones y sus aplicaciones es necesario 'una reorientación específica para adaptar la enseñanza a las necesidades reales de la comunicación académica y profesional' (Aguirre Beltrán, 2012: 5).

En cuanto al español para fines específicos (EFE) tuvo su desarrollo con el auge económico de los años ochenta del siglo pasado, básicamente se trataba del español comercial, sin embargo a partir del ingreso de España en la Comunidad Económica Europea (1986), ha habido un incremento en la demanda del español para fines específicos en el ámbito de la economía tanto en el mundo de los negocios internacionales como otros servicios, entre otros, se encuentran los servicios jurídicos, sanitarios, bancarios, turísticos, etc. (ibid.: 12).

En los últimos años, el español para fines específicos ha tenido que adaptarse a las nuevas necesidades del mercado laboral y profesional siendo éste una 
herramienta fundamental e instrumento de trabajo para ser utilizado en los diferentes sectores de la actividad profesional desde el mundo de los negocios pasando por la industria, la ingeniería, la arquitectura y demás ámbitos, tanto del mundo académico como profesional.

\section{LA LENGUA ESPECIALIZADA}

Tenemos que remitirnos, entre otros, a Pierre Lerat quien con su obra Les langues spécialisées realiza una reflexión global en torno a las lenguas de especialidad, determinando tres aspectos fundamentales y que llevaremos a cabo en este trabajo y en concreto en el ámbito de la arquitectura y de la construcción.

Como fundamento teórico en las lenguas especializadas debemos distinguir como elementos fundamentales entre una morfología léxica adecuada, unas características determinadas de la sintaxis y de la semántica (Lerat, 1997: 8). La finalidad de la lengua especializada según este autor se define como: 'La lengua especializada es ante todo una lengua en situación de empleo profesional, [...] Es la lengua misma como sistema autónomo, pero al servicio de una función más amplia: la transmisión de conocimientos' (ibid.: 18).

A partir de esta definición sobre las lenguas especializadas ya algo alejada en el tiempo aunque igual de válida, nos centraremos en investigaciones más acordes a la realidad actual. Empezaremos con las observaciones de Cassany, quien en algunos de sus trabajos de la enseñanza del español en contextos más especializados, pasó a adoptar sus estudios desde una perspectiva psicolingüística cognitiva en los años noventa a un cambio de orientación más socioculturalcrítica. Cassany se basa en estudios realizados por varios autores en torno a este enfoque:

La perspectiva que adoptan estos estudios se denomina, de manera general sociocultural y se inserta en el viraje que han tenido en los últimos años muchas investigaciones en las ciencias sociales $y$, en particular, en la enseñanza de la lengua. En los últimos años hemos realizado un salto, desde el periodo previo dominado por la psicología y el cognitivismo, hasta una perspectiva que ha redescubierto los aspectos más sociales y socioculturales [...]. (Cassany, 2009: 22)

Este autor propone que la lectura se basa en una práctica letrada, a ello se refiere no sólo como un trabajo mental, sino también sociocultural. Los aspectos socioculturales son muy importantes a la hora de elaborar un texto y en el caso de la obra que vamos a presentar Arquitectura y Construcción. Español académico y profesional, sus autoras hacen especial énfasis en la diversidad cultural tanto en la cultura autóctona española así como en la latinoamericana y sobre todo temas de interés cultural actual para nuestros estudiantes extranjeros.

Otra investigadora, Concha Moreno (2009), partiendo de la lengua de fines específicos propone hablar de contextos profesionales especificos puesto que ello depende de la especificidad del texto y diversifica las diferencias teniendo 
en cuenta los contextos profesionales que van desde la ciencia y la tecnología hasta ámbitos de carácter sociocultural Esta investigadora también parte de una división de los contextos profesionales haciendo hincapié en la enseñanzaaprendizaje y estableciendo un corpus del léxico para cada especialidad teniendo en cuenta los contenidos socioculturales, gramaticales y pragmáticos según las necesidades comunicativas de las diversas necesidades profesionales.

El lenguaje de la ciencia es un lenguaje de tipo formalizado con unas características peculiares, con unas normas terminológicas ya establecidas. Asimismo los textos científicos 'suelen observar las cualidades más esencialmente epistemológicas de la ciencia: universalidad, objetividad, neutralidad (o imparcialidad) y verificabilidad' (Llácer Llorca, 2012: 51). La universalidad juega con el conocimiento del mundo optando normas internacionales como pueden ser las normas DIN, la simbología o bien el Sistema Internacional de Unidades de Medida (SI) (ibid.: 52).

\section{CARACTERÍSTICAS DEL LENGUAJE CIENTÍFICO}

Además en el lenguaje de la ciencia hay que destacar también la precisión y la exactitud. Ambas características son imprescindibles para darle objetividad al texto. En contraposición a otro tipo de lenguajes como podrían ser el lenguaje cotidiano o el lenguaje literario, el lenguaje científico carece de connotaciones afectivas, siempre es imparcial ante unos resultados. La finalidad de todo texto de carácter científico es informar, describir, explicar y argumentar. Las características gramaticales más frecuentes son el uso de oraciones enunciativas, usándose de forma predominante el indicativo como modo de realidad y el uso extendido de adjetivos especificativos. Además el lenguaje científico nos permite emplear el léxico especializado de nuestra lengua, es 'el llamado vocabulario denotativo, aquel que es muy utilizado para conformar taxonomías o clasificaciones' (Becerra, 2002: 22).

Otros recursos que no son propiamente lingüísticos tales como los gráficos, las fórmulas y otros símbolos ayudan a la verificabilidad del texto científico. En el caso del estudio que nos ocupa, en cuanto a la arquitectura y la construcción, 'el lenguaje gráfico es el que supone la mayor dedicación de tiempo y esfuerzo, suponiendo un peso muy importante en los primeros años de estudio' (Castaño y de la Fuente, 2013: 303).

Una vez citadas las características lingüístico-discursivas del lenguaje científico nos centraremos en la aportación léxica y terminológica del lenguaje de la arquitectura y de la construcción.

\section{EL LENGUAJE DE LA ARQUITECTURA Y DE LA CONSTRUCCIÓN}

La arquitectura, como cualquier otra ciencia, dispone de un lenguaje específico a partir de sus orígenes técnicos y se manifiesta a través de los conceptos, del 
vocabulario y estructuras gramaticales específicas. Según Jorge Sainz todo arquitecto usa tres lenguajes. El lenguaje natural para los escritos y la expresión oral, el lenguaje gráfico para dibujos y representaciones gráficas y el lenguaje arquitectónico que consta de una terminología característica (Sainz, 2005, citado en Castaño y de la Fuente, 2013: 17).

El lenguaje de la arquitectura se ha generado con términos a partir del latín, también de la traducción de otras lenguas y se ha apropiado de otras disciplinas científicas, como la medicina tal y como lo afirman Castaño y de la Fuente cuando se habla de patologías de edificios. También es frecuente el uso de prefijos y sufijos cambiando el sentido de las palabras con ejemplos como metaespacios, superlugares. Otros ejemplos derivan de la física y la química como hibridación, o de la biología como células. Por último, se usan también términos adaptados del mundo de la filosofía como hermeneútica, epistemología, holístico etc. (Castaño y de la Fuente, 2013: 304-305).

\subsection{ARQUITECTURA Y CONSTRUCCIÓN. ESPAÑOL ACADÉMICO Y PROFESIONAL}

Nuestra propuesta recomendable se centra en el manual Arquitectura y construcción. Español académico y profesional, diseñado por Paloma Úbeda y María Luisa Escribano. Dicho manual va dirigido a estudiantes y jóvenes profesionales especialistas en arquitectura y construcción. Así es como se define el perfil de dichos estudiantes:

Estos alumnos son estudiantes de arquitectura procedentes de otras universidades europeas, que deben tener superado un nivel básico de español. En ocasiones, asisten a estos cursos con otros estudiantes de la Universidad Politécnica de Madrid que cursan distintas ingenierías (telecomunicación, agrónomos, industriales e informática, son los grupos más numerosos). (Úbeda y Escribano, 2000: 158)

Las autoras hacen hincapié primero en la comunicación representando un escenario o situación cotidiana para el perfil de estudiantes de Arquitectura. En segundo lugar la interacción y palabras en donde los estudiantes presentan una situación real con un contexto delimitado y por último algo de gramática, presentan temas gramaticales centrados en los niveles B1-B2 del Marco Común Europeo de Referencia para las lenguas: Aprendizaje, enseñanza, evaluación (MCER). Asimismo se han tenido en cuenta además de los contenidos gramaticales, los contenidos temáticos y funcionales.

En relación a la Estructura de las unidades constan de tres partes: Texto y contexto; Aspectos culturales; Lápiz y papel. Las tres partes están constituidas en compartimentos estancos cuya finalidad es una Autoevaluación para que el alumno contraste y sepa comunicar sus conclusiones. El manual está orientado de manera que los alumnos lleguen por sí mismos, mediante la interacción, a buscar las soluciones. 
En cuanto al Índice de contenidos está formado por seis unidades didácticas con una descripción de los contenidos muy útiles, según nuestro entender, para todos los estudiantes que se encuentran desplazados a nuestras universidades puesto que la finalidad de los estudiantes es que sepan comunicar sus conocimientos adquiridos y su capacidad de resolución de problemas en entornos nuevos, relacionados con su área de estudio. Así es como lo detallan Úbeda y Escribano en la contraportada:

El manual consta de seis unidades didácticas desarrolladas en cinco escenarios representativos del contexto académico y del ámbito de la arquitectura y la construcción. Cada unidad incluye viñetas motivadoras representativas de cada escenario, diálogos reales, actividades variadas para trabajar la gramática y las distintas destrezas, tareas finales y fichas complementarias de profundización. Además al final del libro aparecen glosarios de terminología universitaria y vocabulario técnico. (Úbeda y Escribano, 2015)

Los blogs de arquitectura (incluidos en las fichas del manual) son materiales audiovisuales muy adecuados para los estudiantes puesto que presentan una mayor contextualización y su finalidad es la comunicación real, esto es, materiales auténticos y su meta es responder a los temas, géneros, léxico y terminología adaptada a las necesidades de la arquitectura y de la construcción.

En definitiva, tal y como apuntan los especialistas: 'La idiosincrasia del lenguaje del arquitecto se manifiesta tanto en los conceptos, como en el vocabulario, y en las estructuras gramaticales' (Castaño y de la Fuente, 2013: 302). En consecuencia, esto implica directamente a los alumnos, (sobre todo en los primeros años de su formación) que necesitan comprender unos códigos de comunicación adecuados para poder estar capacitados y para aprender y usar los términos léxicos adecuados.

A continuación véase un ejemplo (Tabla 1) de representación léxica y terminológica en torno al manual Arquitectura y construcción. Español académico y profesional. Además, incluimos una representación gráfica (Figura 1) del Pequeño diccionario visual de términos arquitectónicos (2018).

Tabla 1 Glosario de términos arquitectónicos

\begin{tabular}{|l|l|l|}
\hline \multicolumn{2}{|c|}{ Glosario de términos arquitectónicos } \\
\hline Español & \multicolumn{1}{c|}{ Inglés } & En tu lengua \\
\hline Bóveda, la & Vault (n) & \\
\hline Dintel, el & Lintel (n) & \\
\hline Fachada, la & Facade (n) & \\
\hline Friso, el & Frieze, dado, baseboard (n) & \\
\hline Lucernario, el & Skylight (n) & \\
\hline Moldura, la & Moulding (n) & \\
\hline
\end{tabular}


Bóveda. Estructura arqueada que cubre el espacio entre dos apoyos y forma el techo o la cubierta de un edificio (Ing.: vault. Fr.: voîte. Al.: Gewölbe. It.: volta).

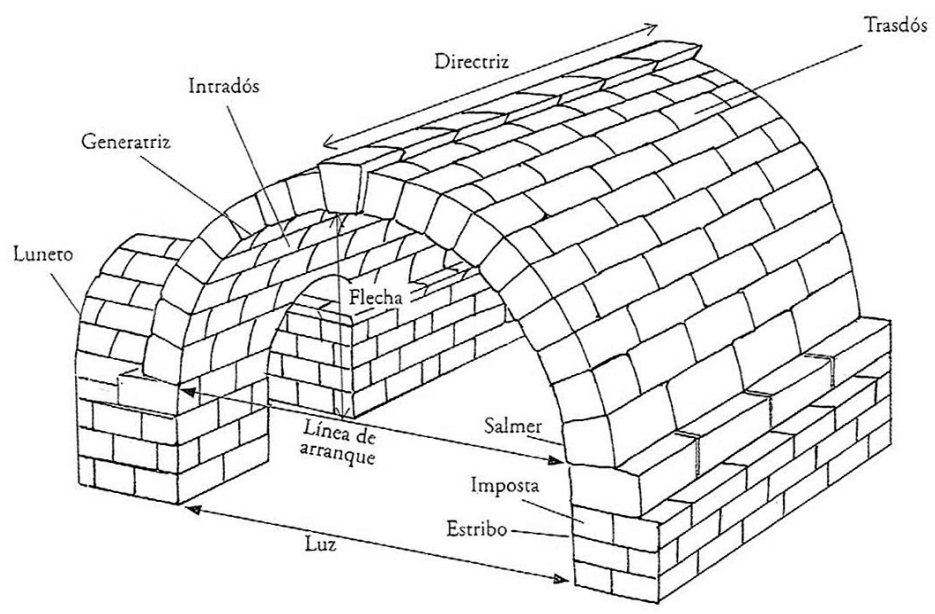

Figura 1 Elementos de la bóveda (De la Plaza Escudero et al., 2018: 45)

\section{CONCLUSIONES}

Con esta comunicación hemos puesto de manifiesto las necesidades de un lenguaje específico para poder lograr los objetivos propuestos y facilitar a los alumnos la comunicación científica adecuada. Debemos valorar lo dicho anteriormente en cuanto a los códigos utilizados, a saber la expresión lingüística afín al lenguaje específico de los arquitectos, la conceptualización de los términos, el uso del léxico apropiado y el lenguaje gráfico. Resumiendo, haremos énfasis a las siguientes características del lenguaje científico ya que debe poseer las siguientes características: especializado, conceptual, preciso, sencillo, lógico y eficaz (Castaño y de la Fuente, 2013: 311).

Después de una serie de observaciones en torno a lo dicho, somos de la opinión en relación al manual Arquitectura y construcción. Español académico y profesional es una herramienta útil de apoyo para nuestros estudiantes, puesto que es una ayuda para el desarrollo de su pensamiento crítico y para mejorar la capacidad de expresión escrita. Debemos resaltar que todos los materiales de enseñanza-aprendizaje tienen que cumplir determinadas cualidades que van desde la motivación pasando por la utilidad, la versatilidad, la accesibilidad y terminando con la adaptabilidad a las diversas necesidades del alumnado. La finalidad debe ser la transmisión del conocimiento y por medio de la estimulación y la motivación, los alumnos pueden desenvolverse en situaciones académicas y profesionales. 
Ello nos lleva a concluir que el manual Arquitectura y construcción. Español académico y profesional es un botón de muestra como herramienta óptima que favorece a los alumnos a conseguir una comunicación fluida. Además ayuda al alumno a usar el lenguaje especializado, a expresarse correctamente por escrito, a desarrollar su pensamiento científico y también a tomar conciencia de los propios errores y las limitaciones de cada uno. Y a propósito del contexto, finalizaremos con una de las sentencias más célebres de Ludwig Wittgenstein: 'los límites de mi lenguaje significan los límites de mi mundo’ (Wittgenstein, 2017: 14).

\title{
REFERENCIAS BIBLIOGRÁFICAS
}

Aguirre Beltrán, B. (2012) Aprendizaje y Enseñanza de Español con fines específicos. Comunicación en ámbitos académicos y profesionales. Madrid: SGEL.

Aguirre, B. (2004) La enseñanza del español con fines profesionales. En J. Sánchez Lobato y I. Santos Gargallo (dir.). Vademecum para la formación de profesores. Enseñar español como segunda lengua (L2) / lengua extranjera (LE) (pp. 109-128). Madrid: SGEL.

Becerra Hiraldo, J. M. (2002) Comentario lexicológico semántico de textos. Madrid: Arco.

Castaño Perea, E. y de la Fuente Prieto, J. (2013) Lenguaje del arquitecto: diagnóstico y propuestas académicas. Revista de Docencia Universitaria REDU, 11 (3): 301-320.

De la Plaza Escudero, L., Morales Gómez, A. y Martínez Murillo, J. M. (2018) Pequeño diccionario visual de términos arquitectónicos. Cuadernos Arte Cátedra. Madrid: Cátedra.

Lerat, P. (1997) Las lenguas especializadas. Ariel: Barcelona.

Llácer Llorca, E. y Ballesteros, F. (2012) El lenguaje científico, la divulgación de la ciencia y el riesgo de las pseudociencias. Quaderns de Filologia. Estudis Lingüístics, 17: 51-67.

Moreno García, C. (2009) De los fines específicos a los contextos profesionales. Disponible en https:// cvc.cervantes.es/ensenanza/biblioteca_ele/asele/pdf/20/20_0129.pdf [Consultado el 2 de marzo de 2019].

Úbeda Mansilla, P. y Escribano Ortega, M. L. (2015) Arquitectura y Construcción. Español académico y profesional. Madrid: Edinumen.

Úbeda Mansilla, P. y Escribano Ortega, M. L. (2000) Algunas pautas para trabajar con textos monográficos en E.L.E.: El campo de español para arquitectos. En Español para Fines Específicos: Actas del I CIEFE (pp. 157-161). Disponible en http://ciefe.com/wp-content/ uploads/2014/05/Actas_I_CIEFE.pdf [Consultado el 29 de marzo de 2019].

Wittgenstein, L. (2017) Tractatus logico-philosophicus-Investigaciones filosóficas (Traducción de I. Reguera Pérez). Madrid: Editorial Gredos.

\section{TEACHING SPANISH FOR SPECIFIC PURPOSES (SSP) AND ITS APPLICATION TO SCIENCE AND TECHNOLOGY}

\begin{abstract}
The Spanish language and Hispanic culture have always aroused great interest across a very broad audience, but in recent years this trend has developed very notably in European universities, not only in the field of humanities but also in many other higher education centers. Spanish has become an optional or obligatory subject in many centers of scientific or technical education, such as polytechnic universities where Spanish plays the role of language for specific purposes and is increasingly considered a language of communication in the academic and professional field. Our work is divided into two thematic
\end{abstract}


areas: on the one hand, the demand for Spanish for Specific Purposes (SSP) as an international communication tool in both academic and professional fields and its application to science and technology, and on the other, architecture and construction, likewise other sciences, constitute in themselves a fundamental product that encompasses industry, technology and knowledge. The author of the paper will show some lexical-terminological aspects and resources in relation to architecture and construction, as well as other characteristics of the scientifictechnical vocabulary.

Key words: Spanish for specific purposes, science, technology, architecture and construction

Isabel Serra Pfennig (Dr. Philol, docente universitaria) trabaja en la Escuela Técnica Superior de Ingenieros Industriales de la Universidad Politécnica de Madrid. Sus investigaciones se centran en la lingüística aplicada a ciencia y a la tecnología. Correo electrónico: isabel.serra@upm.es 